ON THE EXPECTED FREQUENCY OF WR + COMPACT STAR SYSTEMS

D. Vanbeveren and C. de Loore

Astrophysical Institute, Vrije Universiteit Brussel, Brussels, Belgium.

According to the classical evolutionary scenario for massive close binariesit is qenerally assumed that two WR phases are encountered, i.e. a $W R+O B$ stage and a $W R+$ compact star stage. The survival probability of a massive binary after an instantaneous supernova (SN) explosion critically depends on the assumed asymmetry of the SN explosion, i.e. on the "kick" velocity $V_{k}$ (Sutantyo, 1978). The purpose of this paper is to make a statistical study on the expected WR + compact star binaries compared to the WR+OB type binaries based on non-conservative massive binary evolution, as a function of $V_{k}$ which, as we will see, is very poorly known for these types of stars.

\title{
1. THE MODEL
}

- The results of non-conservative massive binary evolution are taken from a series of papers, i.e. Vanbeveren et al. (1979), Vanbeveren and De Grève (1979), Vanbeveren and Packet (1979), Vanbeveren and de Loore (1980), Vanbeveren (1980), Vanbeveren (1981a,b). The conclusions from the latter papers that will be used in the present paper are summarized below.

- Massive close binary evolution has been treated according to a case B mode of mass transfer.

- Effects of overshooting have not been included here.

- During He burning a WR star is losing 40\% of its mass.

- WR stars in close binaries are remnants of primary stars with masses

larger than $40 \mathrm{M}$. If this model is adopted then one has to bear in mind that WR + compact star binaries result from WR+OB systems with $\mathrm{M}_{\mathrm{OB}} \geq 40 \mathrm{MO}$.

- During Roche lobe overflow more than 50\% of the matter leaving the primary also leaves the system.

- Prior to the SN explosion of a primary, the secondary may have reached its own Roche lobe if the initial mass ratio was large enough. This effect has been described by Doom and De Grève (1981); their results are used in the present study. When a secondary star reaches its Roche lobe prior to the SN explosion of the primary, its remnant mass is determined 
using the binary evolutionary computations mentioned earlier.

- For 0 type binaries we have used the mass ratio distribution given by Vanbeveren (1981a).

- During core $\mathrm{H}$ burning a stellar wind mass loss rate is used which removes $\sim 20 \%$ of the ZAMS mass corresponding to $N \simeq 100$ in the formalism of de Loore et al (1978).

- The survival probability $p$ for each system has been computed using the formalism given by Sutantyo (1978). Given the initial mass function by number for primaries with mass larger than $40 \mathrm{M}_{\mathrm{O}}$ (we have used a power function $\psi\left(M_{1}\right) \sim M_{1}-\alpha$ with $\alpha \doteq 1,2$; see also Vanbeveren 1981a) and the normalised mass ratio distribution $\phi(q)$, the mean survival probability during the SN explosion is given by

$$
\overline{\mathrm{P}}=\int_{0.2}^{1} \int_{40}^{100} \mathrm{p} \psi\left(\mathrm{M}_{1}\right) \phi(q) \mathrm{d} \mathrm{M}_{1} \mathrm{dq}
$$

we have taken $100 \mathrm{M}$ as upper mass limit for primary masses; moreover there are very few systems with $q<0.2$.

- If we only consider these binary systems where the secondary mass prior to the first $S N$ explosion is larger than $40 \mathrm{M}_{O}$, we obtain an alternative $q$ distribution $\phi_{1}(q)$. The corresponding $\overline{\mathrm{P}}_{1}$ gives the expected number ratio WR + compact/WR+OB (provided that their evolutionary Iifetimes are the same).

\section{THE RESULTS}

Figure 1 shows $\bar{P}\left(\bar{P}_{1}\right)$ as a function of $v_{k}$ ( $v_{k}=$ the kick velocity relative to the orbital velocity of the exploding star with respect to the

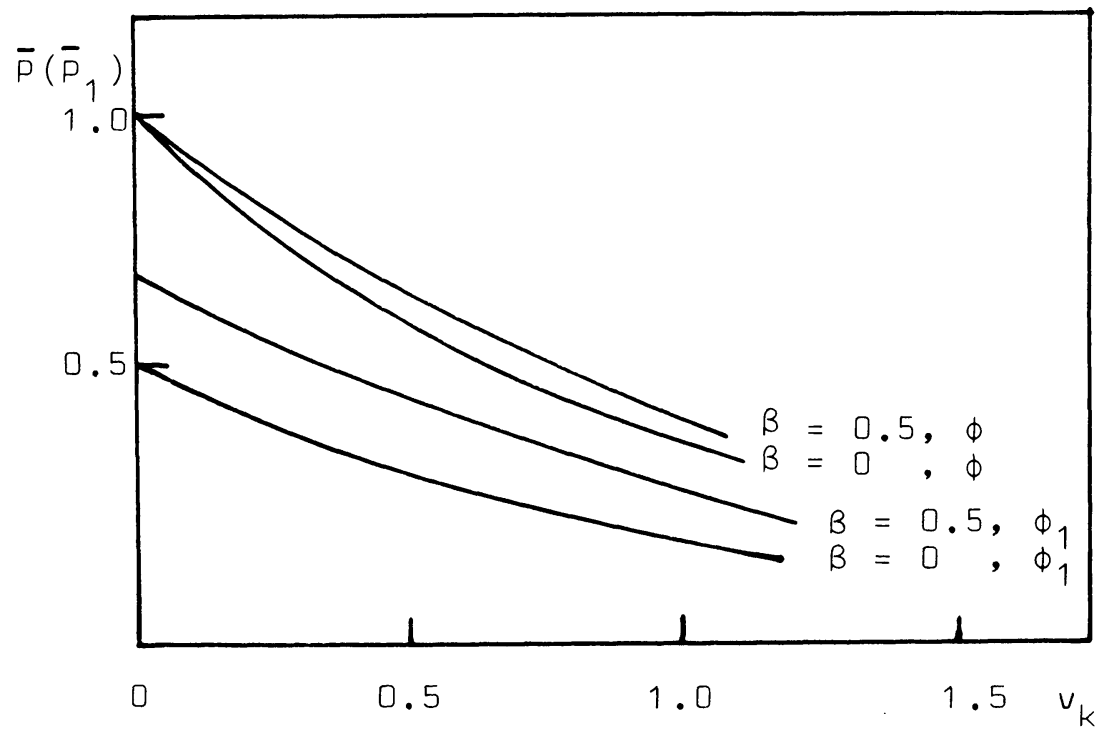

Figure 1. $\bar{P}$ as a function of $v_{k} ; \beta=$ the amount of mass accreted by the secondary. 
companion); different values for the mass loss from the system during Roche lobe overflow are considered. As can be noticed, if $\mathrm{vk}_{\mathrm{k}}=1$ $\left(\mathrm{V}_{\mathrm{k}}=\mathrm{V}_{\mathrm{orb}}\right)$ the mean probability ranges between 0.1 and 0.3 indicating that the expected number of WR + compact star systems shoula be no more than $10 \%$ to $30 \%$ of the number of $\mathrm{WR}+\mathrm{OB}$ systems. What does $\mathrm{v}_{\mathrm{k}}=1$ means for the effective kick velocity $V_{k}$ ? In figure 2 the average kick velocity (computed in the same way as $\overline{\mathrm{P}}$ ) is plotted as a function of the average period for systems prior to the $\mathrm{SN}$ explosion if $\mathrm{vk}_{\mathrm{k}}=1$ and $\mathrm{V}_{\mathrm{k}}=$ 0.5. What do we expect as average period prior to the SN explosion? For the SB2 systems given in the new WR catalogus and taking into account the mass loss during He burning, one finds an average period $\simeq 30$ d. De Loore et al. (1975) investigated SN survival probabilities with $\mathrm{V}_{\mathrm{k}} \simeq 100 \mathrm{~km} / \mathrm{sec}$. They concluded that on the average these probabilities are larger than 0.8 . The difference with our result for $\mathrm{v}_{\mathrm{k}}=0.5$ is due to the fact that we have integrated over a large mass spectrum using more recent mass and mass ratio determinations of $O B$ and $W R$ binaries. Moreover also the $\phi_{1}(q)$ argument plays an important role as can be seen from figure 1. One can ask how far one can believe $V_{k}=100 \mathrm{~km} / \mathrm{sec}$ in the massive star range considered in this study. The transverse velocity of some 20 pulsars is known and ranges between $50 \mathrm{~km} / \mathrm{sec}$ and 600 $\mathrm{km} / \mathrm{sec}$. As many pulsars have originated from single stars, these velocities may reflect the kick velocities gained by the pulsars due to the

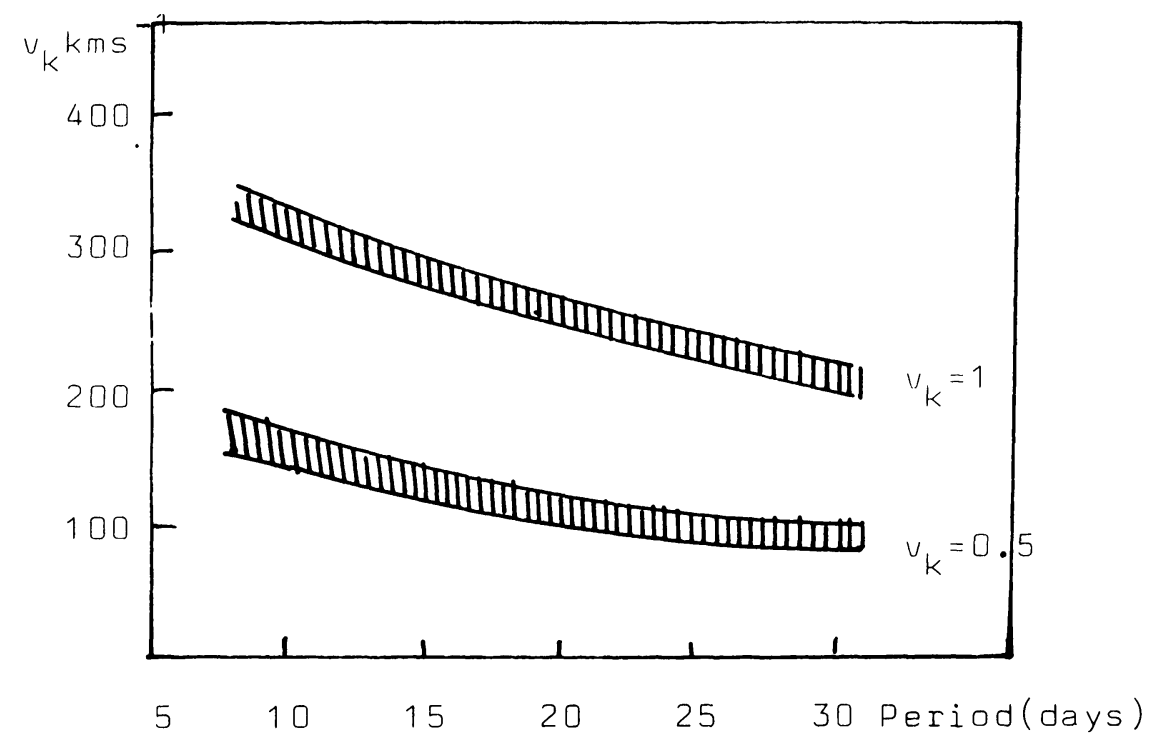

Figure 2. $\quad \overline{\mathrm{V}}_{\mathrm{k}}$ as a function of the period for $\mathrm{V}_{\mathrm{k}}=1$ and for $v_{k}=0.5$.

asymmetric SN explosions. The average transverse velocity is around $260 \mathrm{~km} / \mathrm{sec}$ (taking into account with the sin3i effect, this value increases to about $400 \mathrm{~km} / \mathrm{sec})$. So at first glance $\mathrm{v}_{\mathrm{k}} \geq 1$ or $\mathrm{V}_{\mathrm{k}} \geq 240$ $\mathrm{km} / \mathrm{sec}$ may be not exagerated. It is also a very straightforward exer- 
cise to compute the kick velocity gained by the neutron star as a function of the degree of asymmetry. The velocity of ejection of the $\mathrm{SN}$ shell $\simeq 10^{4} \mathrm{~km} / \mathrm{sec}$ (Shklovsky, 1968); assuming that $10 \%$ of the mass of the SN shell has a velocity which is only 5\% larger (or lower) and assuming $\mathrm{M}_{\text {neutron }}$ star $=2 \mathrm{M}_{\mathrm{O}}$, it follows that for $\mathrm{M}_{\mathrm{Shel}}$ (= mass of the $\mathrm{SN}$ shell) $=8 \mathrm{M}_{\mathrm{O}}, \mathrm{V}_{\mathrm{k}}=200 \mathrm{~km} / \mathrm{sec}$; for $\mathrm{M}_{\mathrm{Shell}}=16 \mathrm{M}_{\mathrm{O}}, \mathrm{V}_{\mathrm{k}}=500$ $\mathrm{km} / \mathrm{sec}$; for $\mathrm{M}_{\text {Shell }}=24 \mathrm{M}_{\mathrm{O}}, \mathrm{V}_{\mathrm{k}}=600 \mathrm{~km} / \mathrm{sec}$; for $\mathrm{M}_{\mathrm{Shell}}=4 \mathrm{M}_{\circ}, \mathrm{V}_{\mathrm{k}}=$ $100 \mathrm{~km} / \mathrm{sec}$. As pulsars from single stars originate from stars with mass larger than $\sim 7 \mathrm{M}_{0}$ (this underlimit may even be lower still), the number of pulsars coming from a star with mass larger than $40 \mathrm{M}_{\circ}$ should be extremely small (within the sample of 20 pulsars there should be at most 1 originating from $M \geq 40 \mathrm{M}_{0}$ ). Again this is an example illustrating how dangerous it can $\bar{b}$ e to use an average value for certain parameters in a whole stellar sample. We therefore formulate the conclusion as follows : saying that there are as many WR + compact star systems as $\mathrm{WR}+\mathrm{OB}$ binaries is wrong. The ratio may be as low as $20 \%$.

\section{REFERENCES}

Doom, C., De Grève, J.P. 1981, Astron.Astrophys., in press.

Heuvel, van den, E.P.J., Heise, J.G. 1972, Nature Phys.Sci. 239, 67.

de Loore, C., De Grève, J.P., Vanbeveren, D. 1978, Astron.Astrophys. 67, 373.

de Loore, C., De Grève, J.P., van den Heuvel, E.P.J., De Cuyper, J.P. 1975, Proc. IAU Regional Meeting, Trieste.

Sutantyo, W. 1978, Astrophys.Space Sci. 54, 479.

Vanbeveren, D. 1981a, Astron.Astrophys. 95, 321.

Vanbeveren, D. 1981b, Astron.Astrophys., in press.

Vanbeveren, D., De Grève, J.P. 1979, Astron.Astrophys. 77, 295.

Vanbeveren, D., de Loore, C. 1980, Astron.Astrophys. 86, 21.

Vanbeveren, D., Packet, W. 1979, Astron.Astrophys. 80, 242.

Vanbeveren, D., De Grève, J.P., van Dessel, E.L., de Loore, C. 1979, Astron.Astrophys. 73, 19.

\section{DISCUSSION FOLLOWING VANBEVEREN AND DE LOORE}

Serrano: One should be very careful when using the IMF. It would seem to me that you should have used the IMF by number rather than by mass, i.e. $\theta \propto \mathrm{M}^{-\alpha-1}$.

Vanbeveren: We have used $\mathrm{M}^{-\alpha}$ with different values for $\alpha$. Adding a-1 in the exponent will not change the conclusions of the present paper. Moreover we have used the results of a recent paper (Astron.Astrophys. 95, 321) where I propose an IMF by number for 0 stars which is different from the one proposed by Lequeux. 
Moffat: Among a complete sample of 14 galactic WNL $(b \leq 12.3)$ stars I mentioned in my review that the ratio of WNL + compact to WNL $+\mathrm{OB}$ is $\simeq 1$ (more precisely 5:3). The rest (6 stars) show no evidence for periodicity. One other star remains unstudied. We have looked for binary evidence with the same intensity for stars at low as at high galactic latitudes. Thus, within small number statistics, this appears to eliminate very high kick velocities.

Vanbeveren: For a theoretician it is very discouraging to see that different observers (Moffat $\leftrightarrow$ Massey) get different conclusions concerning the binary status of a WR star. I have the impression by looking to some velocity curves that a straight line fits the observed points as well. Moreover one should not compare the WNL stages but the whole WR stage because it is not certain that the WNL + OB lifetime is the same as the WNL + compact star lifetime so that the WNL + compact star/ $\mathrm{WNL}+\mathrm{OB}$ ratio does not necessarily reflect a survival probability during the SN explosion. 